\title{
Design of a Segmented LGAD Sensor for the Development of a 4-D Tracking Detector
}

\author{
Sayaka Wada* \\ University of Tsukuba \\ Tsukuba, Ibaraki 3058571, Japan \\ E-mail: wada@hep.px.tsukuba.ac.jp
}

\section{Kyoji Onaru}

University of Tsukuba

Tsukuba, Ibaraki 3058571, Japan

\section{Kazuhiko Hara}

University of Tsukuba

Tsukuba, Ibaraki 3058571, Japan

\section{Yoshinobu Unno}

High Energy Accelerator Research (KEK)

Tsukuba, Ibaraki 3050801, Japan

\section{Koji Nakamura}

High Energy Accelerator Research (KEK)

Tsukuba, Ibaraki 3050801, Japan

\begin{abstract}
Tracking detectors capable of recording the hit-time information at a precision of $\mathrm{O}(10 \mathrm{ps})$ will be breakthrough devices in the future of hadron collider experiments to resolve signal tracks in a harsh environment with significant background tracks. The low-gain avalanche detector (LGAD) is an example of such a device, which is expected to provide real-time fine spatial resolution for a 4-D tracking detector. In this study, we have fabricated LGAD diodes and segmented striptype detectors at Hamamatsu Photonics. We tested pad type detectors using a proton beam and obtained a time resolution better than $30 \mathrm{ps}$ for the devices with an active thickness of $50 \mu \mathrm{m}$. The non-uniformity of the gain across the strip was modeled using TCAD simulation, which was then used to design segmented LGAD sensors with a uniform gain. The study suggests that uniform LGAD sensors can be made with a broader operation bias voltage range. In this paper, we present the test beam and the TCAD simulation results.
\end{abstract}

The 28th International Workshop on Vertex Detectors - Vertex2019

13-18 October, 2019

Lopud, Croatia

${ }^{*}$ Speaker. 


\section{Introduction}

Silicon semiconductor detectors have become essential in many high-energy experiments where precise spatial resolution and fast response are required. The $\mathrm{n}^{+}$-in-p-type sensors, or $\mathrm{p}$-bulk sensors, where fast electrons produce the main part of the signal, are widely used in experiments at the LHC hadron collider as well as the forthcoming HL-LHC. Intensive studies have been carried out to investigate the radiation hardness, and it was found $[1,2]$ that the charge collection is approximately reduced by half at a bias of $500 \mathrm{~V}$ for typical strip sensors irradiated at a fluence of $1 \times 10^{15} 1-\mathrm{MeV} \mathrm{n}_{\mathrm{eq}} / \mathrm{cm}^{2}$. Such a degradation is tolerable for the HL-LHC; however, innovation is required for future experiments where the radiation level will become higher. This implies that the particle density will also become high, which can be partially overcome by reducing the pixel size.

Low-gain avalanche detectors (LGADs), $[3,4,5]$ are a new type of sensors where a $\mathrm{p}^{+}$implant layer added underneath the readout $\mathrm{n}^{+}$electrode provides an internal signal gain due to avalanche multiplication in the region of a high electric field created across the $\mathrm{p}^{+}-\mathrm{n}^{+}$junction. The additional gain can compensate for reduction in the charge collection. Furthermore, LGADs with a moderate gain have shown excellent timing of around 30 ps or faster because a large signal is created during the hole movements in the vicinity of the thin multiplication region. These results encourage us to develop semiconductor detectors with fine spatial and time resolutions, namely 4D detectors.

We examined the LGAD samples fabricated by Hamamatsu Photonics and evaluated their electrical properties [6]. The samples are pad-type detectors with a sensitive area of $1 \mathrm{~mm} \phi$ and strip-type, as shown in Fig. 1. Both types have an active thickness of 50 and $80 \mu \mathrm{m}$, and four different $\mathrm{p}^{+}$doping profiles (A-D), where A-D refers to the doping concentrations in the increasing order.

The timing characteristics were evaluated for the pad-type LGADs using a high-energy proton beam at FNAL, FTBF in February 2018. The gain uniformity of the strip-type was measured [6] by injecting an IR laser, and the data were used to tune the parameters of a TCAD simulation [7]. The simulation was used to design a uniform gain LGAD sensor with segmentation by trenching [8].
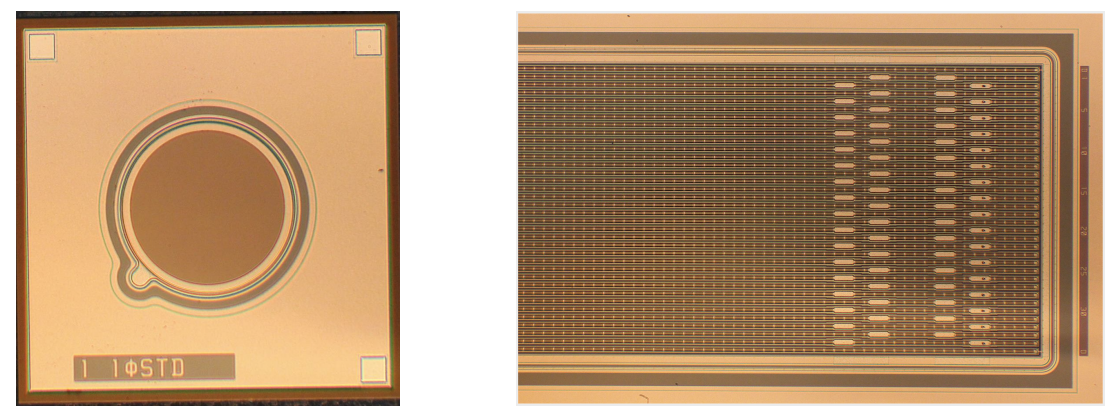

Figure 1: Two types of the evaluated HPK LGAD sensors: pad type (chip size $2.5 \times 2.5 \mathrm{~mm}^{2}$, active area $1 \mathrm{~mm} \phi$ ) and strip type (chip size $6 \times 12 \mathrm{~mm}^{2}$, strip pitch $80 \mu \mathrm{m}$ ) 


\section{Evaluation of timing precision in high-energy beam}

\subsection{Beam test setup}

Three pad-type LGADs with thicknesses 50 and $80 \mu \mathrm{m}$ were stacked along the $120-\mathrm{GeV}$ proton beam at Fermilab. The sensors were enclosed in a Styrofoam box to control the temperature inside; the temperature was lowered by placing blocks of dry ice on the box. The signals were amplified by fast discrete amplifiers [9] of $75-\mathrm{GHz}$ bandwidth and a gain of 100, then digitized using fast FADCs, CAEN V1742 based on DRS4 technology [10]. The pulse shape was obtained in 12 bits (1 Vpp) at 5-GHz sampling for a total duration of $200 \mathrm{~ns}$. The event trigger was provided by two sets of scintillation counters and an ATLAS FE-I4 based pixel module [11] which restricts the beam area by setting the region of interest.

\subsection{LGAD signal}

The maximum voltages (absolute values) in correlation with the beam are plotted on a histogram in Fig. 2. A cluster of events with large pulses with the lvoltagel $>40 \mathrm{mV}$ is eveident corresponding to the tracks traversing the gain region. Over the pedestal (below $5 \mathrm{mV}$ ), the cluster below $40 \mathrm{mV}$ is for the tracks traversing the peripheral region with no LGAD gain. For those events with lvoltagel $>40 \mathrm{mV}$, the signal shapes of the peak are also shown in Fig. 2.
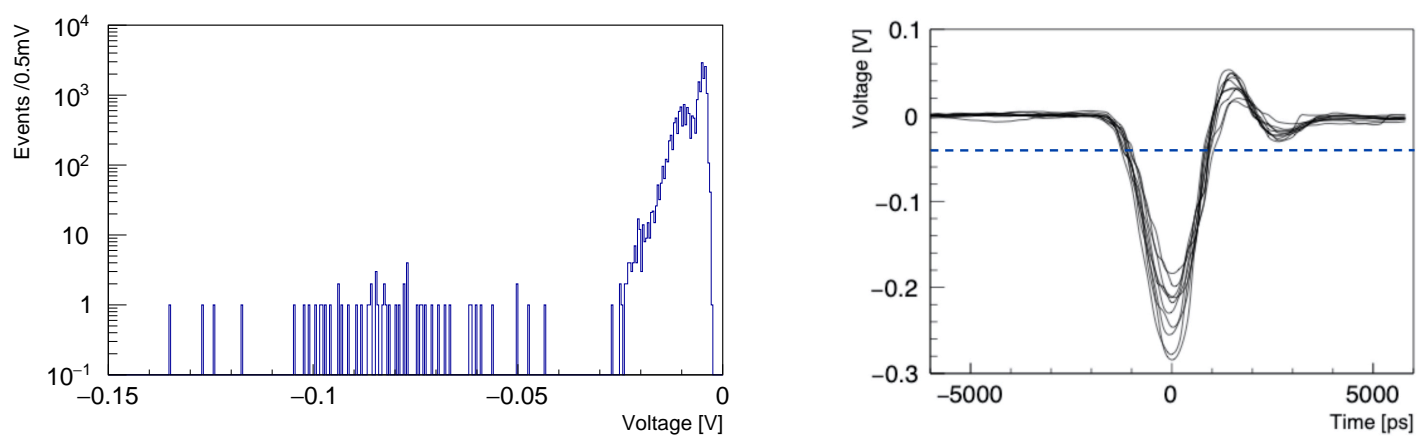

Figure 2: Pad-type LGAD response measured with the FNAL test beam; (left) distribution of the maximum voltages in correlation with the beam, (right) signal shapes for the events with a maximum voltage greater than $40 \mathrm{mV}$.

\subsection{Time resolution}

\subsubsection{Evaluation methods}

The time resolution achievable in typical practical electronics was evaluated from the pulse shapes given in Fig. 2. As the time walk deteriorates the time resolution, the time exceeding a single threshold is not precise enough. Here, we investigated the time resolution based on a constant fraction method where the time is given at a voltage exceeding a certain fraction of the peak voltage. The measured pulse shape was fitted to a quintic function. The peak pulse height $V_{\text {peak }}$ was then calculated and the time at a voltage exceeding $f \times V_{\text {peak }}$ was derived, where $\mathrm{f}$ stands for the constant fraction. 
The same method was applied to the three pad-type LGADs in the beam. As the two sensors named 1 and 2 had a similar active thickness of $50 \mu \mathrm{m}$, the event-by-event time difference $T_{1}-T_{2}$ shows a Gaussian distribution with a standard deviation given by

$$
\sigma\left(T_{1}-T_{2}\right)=\sqrt{\sigma_{1}^{2}+\sigma_{2}^{2}} \sim \sqrt{2} \sigma
$$

where $\sigma$ is the intrinsic time resolution of the LGAD \#1 and \#2. The time resolution for LGAD \#3 with an active thickness of $80 \mu \mathrm{m}$ was similarly derived from the $T_{3}-T_{2}$ distribution and the previously measured $\sigma_{2}$ value.

\subsubsection{Results of the time resolution}

Figure 3 shows plots of the evaluated time resolutions as a function of the fraction $f$. The results are given for 50 and $80 \mu \mathrm{m}$ active thicknesses and at different temperatures. Generally, the time resolution reaches a minimum for the fraction $f>0.3$. The resolution is better for the LGAD with an active thickness of $50 \mu \mathrm{m}$ than that with $80 \mu \mathrm{m}$. The resolution improved and the gain increased by reducing the temperature.
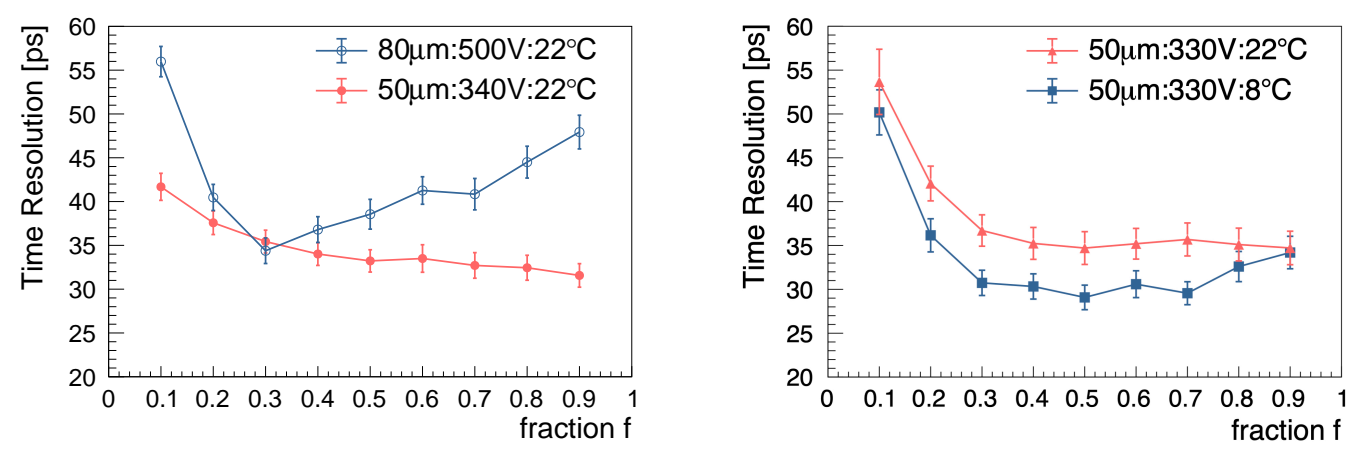

Figure 3: Evaluated intrinsic time resolution of the pad-type LGAD sensors: (left) 50 and $80 \mu \mathrm{m}$ active thicknesses, (right) $50 \mu \mathrm{m}$ active thickness measured at 22 and $8^{\circ} \mathrm{C}$. The horizontal is the ratio of the threshold voltage to the peak voltage (see text).

\section{TCAD simulation for segmented LGADs}

\subsection{Validation of TCAD parameters from comparison with measured characteristics}

TCAD is a device simulator commonly used in semiconductor design, and we developed a simulation to represent the LGAD characteristics. The parameters, such as depth and lateral doping profiles, are essential in the adoption of TCAD. After iterative trials by introducing best guesses, the TCAD can reproduce the general trend as a function of the bias of the leakage current (I-V) and the bulk capacitance (C-V) of a pad-type LGAD, as shown in Fig. 4 and Fig. 5. The precise reproduction of the measurements is not complete because of the simplified lateral profiles, but the breakdown voltages are well described. Moreover, the simulation can reproduce the response profile in the strip-type detector, as shown in Fig. 6. Here the response across the strip was measured by injecting a pulsed IR laser, which was collimated to $2 \times 2 \mu \mathrm{m}^{2}$ (some missing points are due to 
the $\mathrm{Al}$ electrode). From the overall results presented here, we were encouraged to extend the TCAD to simulate the response of the LGAD that was segmented by trenches to evaluate the response uniformity and the breakdown characteristics.
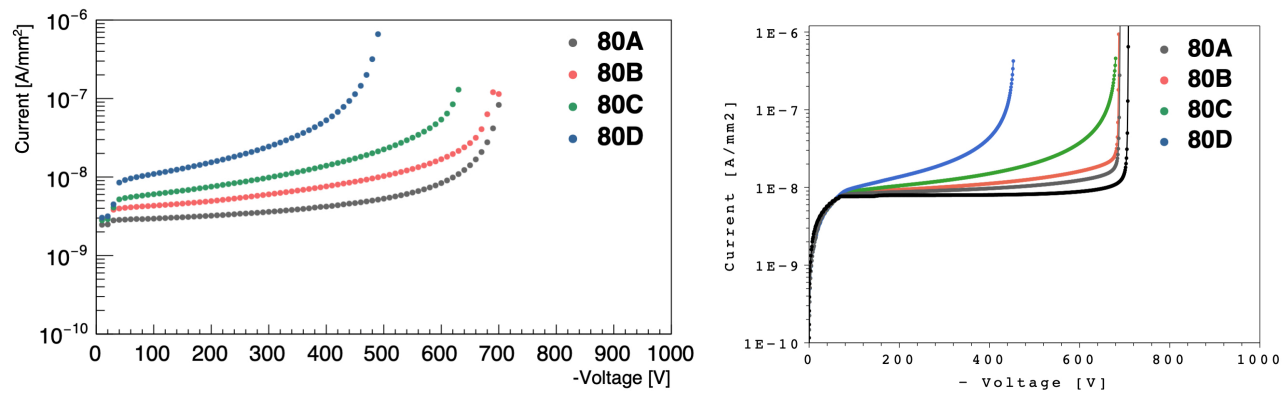

Figure 4: I-V curves of the pad-type LGAD sensors ( $80 \mu \mathrm{m}$ active thickness and four different $\mathrm{p}^{+}$implant concentrations): (left) measurement, (right) TCAD simulation. The simulation includes the estimation for the PIN diode (no amplification) in black.
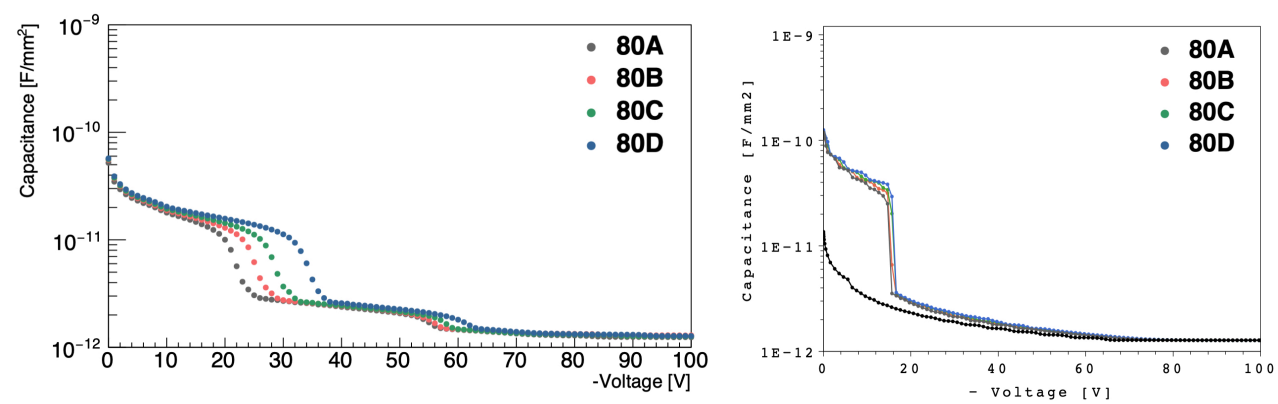

Figure 5: $\mathrm{C}-\mathrm{V}$ curves of the pad-type LGAD sensors ( $80 \mu \mathrm{m}$ active thickness and four different $\mathrm{p}^{+}$implant concentrations): (left) measurement, (right) TCAD simulation. The simulation includes the estimation for the PIN diode (no amplification) in black.
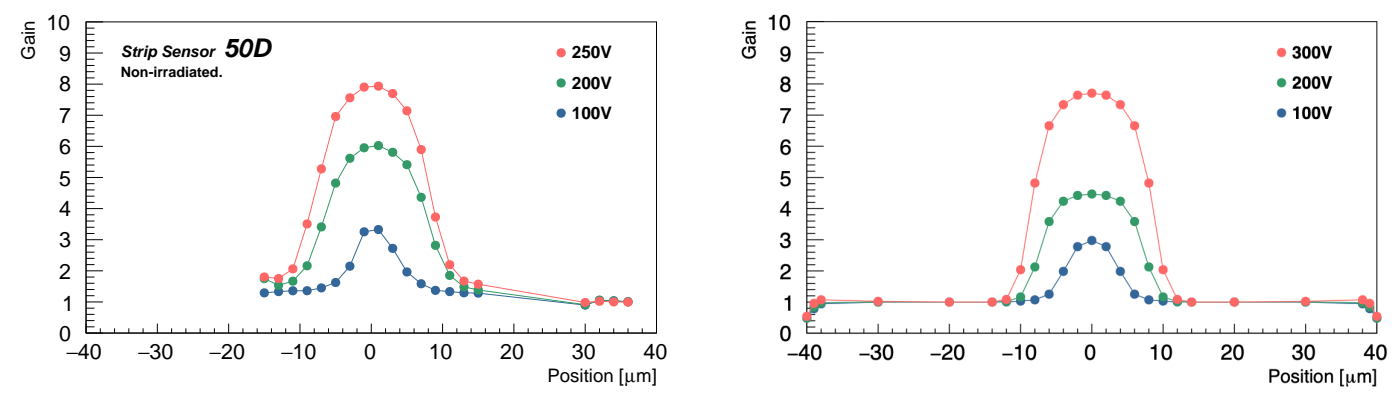

Figure 6: Gain distributions across the strip at different bias voltages; (left) measurement by injecting collimated IR laser, (right) TCAD simulation.

\section{TRENCH structure as a candidate for uniform gain}

The readout $\mathrm{n}^{+}$implant and the $\mathrm{p}^{+}$implant underneath have a uniform lateral doping, and the readout electrodes are separated physically by deep trenches [8]. Such an LGAD was simulated 
with the TCAD. Figure 7 illustrates the impact ionization profile, namely the multiplication profile, across the trenches located at 40 and $120 \mu \mathrm{m}$. The largest multiplication is located (shown in red) under the readout electrode (in blue) and it extends to the vicinity of the trenches. The trench width is $3 \mu \mathrm{m}$ and depth is $5 \mu \mathrm{m}$. The electric field is uniform with a vertical direction. The field around the trench is significant, and this point initiates the breakdown.

The breakdown and response uniformity are shown in Fig. 7. The breakdown characteristics are compared with the strip-type LGAD and the behavior of the trench is milder than that of the strip type. The response is uniform to the vicinity of the trenches.

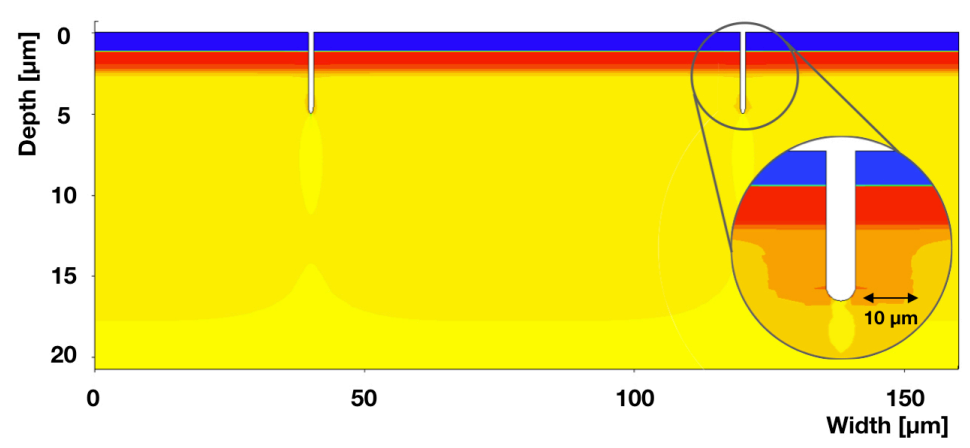

Figure 7: Impact ionization distribution shown in a cross section of the trenched LGAD (TCAD simulation). Enlarged is the detail around the trench.
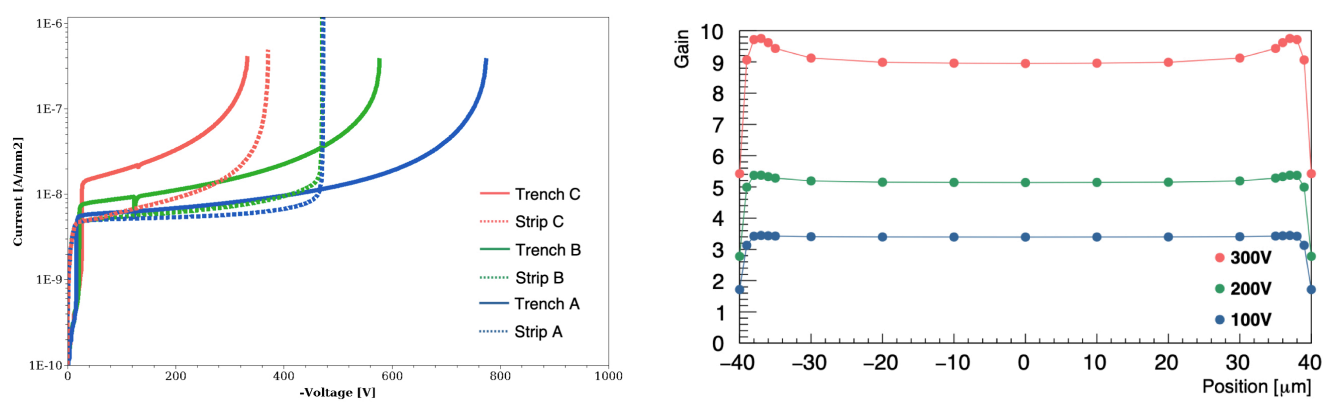

Figure 8: (left) I-V curves for the LGADs segmented by the trench (solid) and the strip (dotted) shown for three $\mathrm{p}^{+}$concentrations. (right) The gain uniformity at different bias voltages. The trench is located at $\pm 40 \mu \mathrm{m}$.

\section{Conclusions}

We are developing segmented LGAD sensors for a realization of a 4D tracker. Pad-type LGADs with $50 \mu \mathrm{m}$ active thickness showed a time resolution of $30 \mathrm{ps}$. A TCAD simulation was developed to reproduce the measured LGAD characteristics, which was used to design a segmented LGAD with uniform gain. The trench separation was suitable to achieve effective segmentation.

\section{Acknowledgements}

This work was supported in part by JSPS KAKENHI Grant Number 19H04393 and 19H05193, 
and U.S.-Japan Science and Technology Cooperation Program in High Energy Physics (FY20182019). The discussions with K. Yamamura and S. Kamata of Hamamatsu Photonics were very inspiring. We appreciate the support provided by the FTBF operation group, led by M. Rominsky. Cooperation with A. Apresyan of FNAL has been very fruitful, and his support was vital in carrying out the test beam measurements.

\section{References}

[1] K. Hara et al., Nucl. Instrum. Methods A 831 (2016) 181-188.

[2] V. Cindro et al., Nucl. Instrm. Methods A 924 (2019) 153-159.

[3] I. Tappen et al., Nucl. Instrm. Methods A 388 (1997) 79-90.

[4] G. Pellegrini et al., Nucl. Instrm. Methods A 765 (2014) 12-16.

[5] N. Cartiglia et al., JINST, 9 (02) (2014), p. C02001.

[6] S. Wada et al., Nucl. Instrum. Methods A 924 (2019) 380-386.

[7] TCAD synopsis, homepage: https://www.synopsys.com/

[8] G. Borghi et al., "Development and First Parametric Characterization of High Fill-Factor Segmented LGADs with Trench Isolation”, 34th RD50 Workshop, Lancaster, June 12, 2019.

[9] A. Apresyan, private communication.

[10] S. Ritt, IEEE Nuclear Science Symposium 2008 Oct. 19th-25th, 2008, Dresden, Germany.

[11] M. Garcia-Sciveres et al, Nucl. Instrum. Methods A 636 (2011) S155-S159. 